\title{
EXTREMAL STRUCTURE OF WELL-CAPPED CONVEX SETS
}

\author{
BY \\ L. ASIMOW( $\left.{ }^{1}\right)$
}

Introduction. The Krein-Milman Theorem states that a compact convex subset of a locally convex topological vector space (LCTVS) is the closed convex hull of its extreme points. Klee [5] has generalized this by proving that if $X$ is a locally compact closed convex subset of a LCTVS, and $X$ contains no line, then $X$ is the closed convex hull of its extreme points and extremal rays.

Another approach to extending the Krein-Milman Theorem, due to Choquet [1], uses the concept of a cap of a convex cone. A set $C$ is said to be a cap of the cone $P$ if $C$ is a compact convex subset of $P$ for which $P \backslash C$ is also convex. If each point of $P$ is contained in a cap, then $P$ is said to be well-capped. Choquet showed that if a closed convex cone in a LCTVS is well-capped, then it is the closed convex hull of its extremal rays.

These two approaches can be unified by extending the definition of cap to apply to arbitrary closed convex sets. Thus a cap $C$ of a closed convex set $X$ is a compact convex subset of $X$ such that $X \backslash C$ is convex. The set $X$ is well-capped if it the union of its caps. Klee's techniques in [5] can be applied to show that a locally compact closed convex set containing no line is well-capped and that any closed convex well-capped subset of a LCTVS is the closed convex hull of its extreme points and extremal rays.

It follows from the above that the statement of a Krein-Milman type theorem for well-capped sets includes the Klee and Choquet versions simultaneously. This is the principal motivation for the study of well-capped sets. It will be shown that each closed convex well-capped set generates (in a canonical way) a closed convex well-capped cone. In this sense the class of well-capped sets is closely related to the class of well-capped cones. A detailed discussion of well-capped cones can be found in [8].

In $\S 1$ some of the basic algebraic facts about caps of arbitrary closed convex sets are discussed. The notion of the cap boundary is introduced and is used to provide a convenient description of a cap by means of a certain affine functional.

In $\S 2$ the properties of well-capped sets and cones mentioned above are developed. $\$ 3$ deals with various positive cones of measures on a locally compact Hausdorff space, and criteria for a measure to be contained in a cap are given. Meyer [7] has shown that if $T$ is a locally compact, $\sigma$-compact Hausdorff space, with $C_{\infty}(T)$

Received by the editors March 13, 1967 and, in revised form, May 10, 1968.

( ${ }^{1}$ Supported in part by the National Science Foundation. 
the space of continuous functions on $T$ with compact support, then the cone of positive regular Borel measures on $T$ is well-capped in the weak topology induced by $C_{\infty}(T)$. It is shown that if $\mu$ is a positive regular Borel measure on the locally compact Hausdorff space $T$ such that either the support of $\mu$ is $\sigma$-compact or $\mu(T)$ is finite, then $\mu$ is contained in a cap of the cone of positive regular Borel measures. It is shown further that if $T$ is a paracompact, locally compact Hausdorff space then $\mu$ is contained in a cap if and only if the support of $\mu$ is $\sigma$-compact.

$\S 4$ contains examples of closed convex sets which fail to be well-capped. Theorem 4.1 shows the existence of a closed convex set which is not well-capped, but is the vector sum of a universally capped cone (a cap $C$ of $P$ is called a universal cap of $P$ if $P=\bigcup_{n=1}^{\infty} n C$ ) and a compact convex set. Thus the property of being well-capped is not preserved under set addition, even in this weak form. The remainder of the section is devoted to showing that several of the standard ordered topological vector spaces have positive cones which fail to be well-capped. They include the $L^{p}$ spaces, $1<p \leqq \infty$, in their weak* topologies, and some of the frequently encountered function spaces.

We wish to acknowledge our indebtedness to Professor R. R. Phelps for his many valuable suggestions during the preparation of the thesis from which this paper is drawn.

1. Let $E$ be a Hausdorff locally convex topological vector space (LCTVS). The following notation will be used throughout: $(x, y)$ denotes the open interval connecting the points $x$ and $y$ in $E,[x, y)$ the closed-open interval, etc. Let $) x, y($ denote the line in $E$ determined by $x$ and $y$. The open ray determined by $x$ and $y$ with end-point $x$ is denoted by $(x, y$ ( and the corresponding closed ray is denoted by $[x, y($.

Let $X$ be a closed convex subset of $E$.

Definition. A subset $S$ of $X$ is extremal if whenever $x, y \in X$ and $(x, y) S \neq \phi$, then $[x, y] \subset S$.

In working with well-capped sets, it is of considerable technical convenience to introduce a certain affine functional associated with a given cap. The purpose of this section is to construct this functional (see Theorem 1.6).

Definition. Let $C$ be a cap of $X$. The cap boundary $B_{C}$ of $C$ in $X$ is $\{x \in C \mid$ there exist $x_{1}, x_{2}$ such that $\left.x \in\left(x_{1}, x_{2}\right) ; x_{1} \in C,\left(x, x_{2}\right] \subset X \mid C\right\}$.

It is readily seen that $B_{C}$ is convex and that $B_{C}$ is empty if and only if $C$ is extremal in $X$. Let $S_{C}$ denote the affine variety spanned by $C$. If $B_{C}$ is not empty let $L_{C}$ be the affine variety spanned by $B_{C}$. Since $C$ and $B_{C}$ are convex,

$$
S_{C}=\bigcup\left\{\left(x, y(\mid x, y \in C\} \text { and } L_{C}=\bigcup\left\{\left(x, y\left(\mid x, y \in B_{C}\right\} .\right.\right.\right.\right.
$$

Proposition 1.1. (i) If $C$ is an extremal cap of $X$, then $S_{C} \cap X=C$.

(ii) If $C$ is any cap of $X$, then $S_{C} \cap X$ is extremal in $X$.

(iii) If $S$ is an extremal subset of $X$ and $C$ is a cap of $S$, then $C$ is a cap of $X$. In particular if $C$ is a cap of $X$ and $C^{\prime}$ is a cap of $S_{C} \cap X$, then $C^{\prime}$ is a cap of $X$. 
Proof. (i) If $x \in\left(S_{C} \cap X\right) \mid C$ then $x \in(y, z(; y, z \in C$. But then $z \in(y, x)$ so that $B_{C} \neq \phi$.

(ii) If $C$ is extremal, the conclusion follows from (i). Suppose that $B_{C} \neq \phi$ and that $x \in\left(x_{1}, x_{2}\right) \cap S_{C} ; x_{1}, x_{2} \in X$. If $x \in C$ then either $x_{1}$ or $x_{2}$, say $x_{1}$, is in $C$. But then $x_{2} \in\left(x_{1}, x\left(\subset S_{C}\right.\right.$, so $x_{1}, x_{2} \in S_{C}$. If $x \in\left(S_{C} \cap X\right) \mid C$ then $x \in(y, z(; y, z \in C$. Since $x \in\left(x_{1}, x_{2}\right)$ and $z \in(y, x)$, we have $z \in\left(z_{1}, z_{2}\right)$ where $z_{i} \in\left(y, x_{i}\right) ; i=1,2$. But then $z \in C$ implies $z_{1}$ and $z_{2}$ are in $S_{C}$. Thus $x_{i} \in\left(y, z_{i}\left(\subset S_{C}\right.\right.$.

(iii) Suppose $x_{1}$ and $x_{2}$ are in $X \mid C$. If $\left(x_{1}, x_{2}\right) \cap C \neq \phi$ then $x_{1}, x_{2} \in S$ since $S$ is extremal. But $S \backslash C$ is convex so that either $x_{1}$ or $x_{2}$ is in $C$. Thus $\left[x_{1}, x_{2}\right] \subset X \backslash C$.

Assume now that $C$ is a cap of $X$ and that the cap boundary $B_{C}$ is not empty.

LEMMA 1.2. Suppose $z$ is contained in $C$.

(i) If $z \in L_{C}$, then $(z, y] \subset X \mid C$ for all $y \in X \mid C$.

(ii) If $z \in C \mid L_{C}$, then $(z, y) \cap B_{C} \neq \phi$ for all $y \in\left(S_{C} \cap X\right) \mid C$.

Proof. (i) Assume first $z \in B_{C}$ and without loss of generality take $z=0$. Thus there is an $x_{1} \in C$ such that $\left(0,-x_{1}\right] \subset X \mid C$. Given $y \in X \mid C$ we must show that $\lambda_{0} y \in X \mid C ; 0<\lambda_{0}<1$. There exists an $\eta_{0}, 0 \leqq \eta_{0}<1$, such that $\eta y+(1-\eta) x_{1} \in X \mid C$ for all $\eta, 1 \geqq \eta>\eta_{0}$. Then if $x=\eta y+(1-\eta) x_{1}$ with $1>\eta>\eta_{0}$ and $\eta$ sufficiently close to $1, x \in X \mid C$ and the ray $\left(x, \lambda_{0} y\right.$ (intersects $\left(0,-x_{1}\right)$ at some point $w$. Then $\lambda_{0} y$ $\in(x, w) ; x, w \in X \mid C$, so that $\lambda_{0} y \in X \mid C$.

If $z=0 \in L_{C} \mid B_{C}$ then there exist $x, \eta(0<\eta<1)$ such that $x, \eta x \in B_{C}$. It is easy to verify that there exists $w \in\left(x, \lambda_{0} y\right) \cap(\eta x, y)$. Since $\eta x \in B_{C}, w \in X \mid C$. By the convexity of $C, \lambda_{0} y \in X \backslash C$.

(ii) Again take $z=0$. Since $y \in S_{C}, y \in\left(x_{1}, x_{0}\left(; x_{1}, x_{0} \in C\right.\right.$. Since $y \in X \backslash C, x_{0}$ can be chosen to be in $B_{C}$. It suffices to show there exists $\lambda_{0}\left(0<\lambda_{0}<1\right)$ for which $\lambda_{0} y \in C$. Suppose not, so that $(0, y] \subset X \mid C$. There exists a $w \in(0, y)$ such that $x_{0} / 2 \in\left(w, x_{1}\right)$. Furthermore, if $w^{\prime} \in\left(x_{0} / 2, w\right)$, then $w^{\prime} \in\left(x_{0}, \lambda y\right)$ for some $\lambda, 0<\lambda<1$. Thus by (i) $w^{\prime} \in X \backslash C$. Thus $x_{0} / 2 \in B_{C}$. But $x_{0}$ is in $B_{C}$ also so that this implies $0 \in L_{C}$. Thus $\lambda_{0} y \in C$ for some $\lambda_{0}, 0<\lambda_{0}<1$.

Corollary 1.3. If $z \in L_{C} \cap X$, then $z \in C$ and hence $L_{C} \cap X=L_{C} \cap C$.

Proof. This follows from (i) and the fact that $L_{C}=\bigcup\left\{\left(x, y\left(\mid x, y \in B_{C}\right\}\right.\right.$.

Corollary 1.4. The sets $C \mid L_{C}$ and $\left(S_{C} \cap X\right) \mid C$ are nonempty.

Proof. This follows from (i) and the fact that $B_{C}$ is assumed to be nonempty.

Corollary 1.5. Assume $0 \in C \mid L_{C}$. Then $S_{C}=\left\langle L_{C}\right\rangle$, the linear subspace generated by $L_{C}$, and hence $L_{C}$ is a hyperplane in $S_{C}$ disjoint from the origin.

Proof. Clearly $\left\langle L_{C}\right\rangle \subset S_{C}$. To show the reverse inclusion it suffices to show $C \subset\left\langle L_{C}\right\rangle$. If $x \in C \cap L_{C}$, then $x \in\left\langle L_{C}\right\rangle$. If $x \in C \mid L_{C}$, then by (ii) there exists $x_{0}, y$ such that $x_{0} \in B_{C}, y \in\left(S_{C} \cap X\right) \backslash C$ and $x_{0} \in(x, y)$. Also by (ii) there is an $x_{1} \in(0, y)$ $\cap B_{C}$. Thus $x_{0}=\lambda x+(1-\lambda) y ; 0<\lambda<1$, and $x_{1}=\mu y ; 0<\mu<1$. Then

$$
x=x_{0} / \lambda-[(1-\lambda) / \lambda] y=x_{0} / \lambda-[(1-\lambda) / \lambda]\left(x_{1} / \mu\right) \in\left\langle L_{C}\right\rangle .
$$


We now formulate the main result of this section.

THEOREM 1.6. Let $C$ be a cap of $X$ with a nonempty cap boundary (equivalently, $C$ is not extremal in $X$ ) and let $S_{C}$ be the affine variety spanned by $C$. There exists an affine functional $f$ on $S_{C}$ such that

(i) $C=\left\{x \in S_{C} \cap X \mid f(x) \leqq 1\right\}$,

(ii) $C_{r}=\left\{x \in S_{C} \cap X \mid f(x) \leqq r\right\}$ ( $r$ any real number) is either empty or a cap of $X$,

(iii) $f$ is lower-semicontinuous on $S_{C} \cap X$,

(iv) $f$ is bounded below on $S_{C} \cap X$ and $C^{\prime}=\left\{x \in S_{C} \cap X \mid f(x)=\inf f\left(S_{C} \cap X\right)\right\}$ is a nonempty extremal cap of $X$ contained in $C \mid L_{C}$.

(v) If $g$ is any other affine functional on $S_{C}$ satisfying (i) then $g=\alpha f+(1-\alpha)$, where $\alpha>0$. Thus the family of caps $\{C\}_{r \in R}$ and in particular the cap $C^{\prime}$ (as in (iv)) is independent of the choice of $f$.

(vi) If $0 \in C \mid L_{C}$, then $S_{C}$ is a subspace and there is a unique linear functional on $S_{C}$ satisfying (i); if $0 \in C^{\prime}$, then there exists a unique linear functional on $S_{C}$ bounded below by 0 on $S_{C} \cap X$ and satisfying (i).

Proof. Corollary 1.4 guarantees that $C \mid L_{C}$ is not empty. It is easiest to consider first the case where $0 \in C \mid L_{C}$. By Corollary 1.5, there is a unique linear functional $f$ on $S_{C}$ such that $f^{-1}(1)=L_{C}$. Choose $x_{0} \in B_{C}$ and $\lambda_{0}>1$ such that $\left(x_{0}, \lambda_{0} x_{0}\right] \subset X \mid C$. To show $f$ satisfies (i), let $x \in C$. If $x \in C \mid L_{C}$, there exists (by 1.2) $z \in B_{C}$ such that $z=(1-\lambda) x+\lambda\left(\lambda_{0} x_{0}\right), 0<\lambda<1$. Since $z, x_{0} \in L_{C}$,

$$
f(x)=f\left[\left(z-\lambda \lambda_{0} x_{0}\right) /(1-\lambda)\right]=\left(1-\lambda \lambda_{0}\right) /(1-\lambda)<1 .
$$

Thus $C \subset\left\{x \in S_{C} \cap X \mid f(x) \leqq 1\right\}$. Now let $x \in S_{C} \cap X$ and suppose $f(x) \leqq 1$. To show $x \in C$ it suffices to show that $\left[x, \lambda_{0} x_{0}\right) \cap L_{C} \neq \phi$. We have $x=r x_{0}+y$, where $r \leqq 1$ and $f(y)=0$. If $\alpha=\left(\lambda_{0}-1\right) /\left(\lambda_{0}-r\right)$, then $0<\alpha \leqq 1$ and $x_{0}+\alpha y=\alpha x+(1-\alpha)\left(\lambda_{0} x_{0}\right)$. Since $f\left(x_{0}+\alpha y\right)=1, x_{0}+\alpha y \in L_{C}$. Thus $x_{0}+\alpha y \in L_{C} \cap X=L_{C} \cap C$.

To prove (ii) it suffices to show $C_{r}$ is compact, for then the linearity of $f$ and Proposition 1.1 serve to show $C_{r}$ is a cap of $X$. Suppose first $r \geqq 1$. Then it is easily seen that $C_{r}=(r C) \cap X$. If $r<1$, then $C_{r}=\left[\lambda_{0} x_{0}+\lambda\left(C-\lambda_{0} x_{0}\right)\right] \cap X$, where $\lambda=$ $\left(\lambda_{0}-r\right) /\left(\lambda_{0}-1\right)$. In either case $C_{r}$ is compact.

Since $C_{r}=\left\{x \in S_{C} \cap X \mid f(x) \leqq r\right\}$ is compact it follows that $f$ is l.s.c. on $S_{C} \cap X$. This proves (iii).

Since $f$ is l.s.c. on the compact set $C, f$ assumes its greatest lower bound at some point $x \in C$. Say $f(x)=a$. Since $0 \in C, a \leqq 0$. If $y \in S_{C} \cap X$ and $f(y) \leqq a$ then $y \in C$ and hence $f(y)=a$. Thus $a=\inf f\left(S_{C} \cap X\right)$. Now $C^{\prime}=\left\{x \in S_{C} \cap X \mid f(x)=a\right\}$ is seen to be an extremal cap of $X$ and since $a \leqq 0, C^{\prime} \subset X \mid L_{C}$. If $0 \in C^{\prime}$, then $f$ is bounded below on $S_{C} \cap X$ by 0 .

To show (v) suppose $g$ is an affine functional on $S_{C}$ satisfying (i). If $x \in B_{C}$, then $g(x) \leqq 1$. But $x=\left(x_{1}+x_{2}\right) / 2 ; x_{1} \in C$ and $\left(x, x_{2}\right] \in X \backslash C$. So, if $g(x)<1$ then $\left(x, x_{2}\right]$ $\cap\left\{x \in S_{C} \cap X \mid g(x) \leqq 1\right\} \neq \phi$. Thus $g(x)=1$ and hence $g\left(L_{C}\right)=\{1\}$. If $x \in C \mid L_{C}$ it 
follows from Lemma 1.2 (ii) that $g(x)<1$. Thus $g^{-1}(1)=f^{-1}(1)=L_{C}$ so that $g=$ $\alpha f+(1-\alpha)$. Since $\left\{x \in S_{C} \mid g(x) \leqq 1\right\}=\left\{x \in S_{C} \mid f(x) \leqq 1\right\}, \alpha>0$.

This completes the proof in case $0 \in C \mid L_{C}$. The general case follows easily by considering an appropriate translation of $X$.

Definition. If $C$ is a cap of $X$ with $B_{C} \neq \phi$ then any $f$ satisfying (i) will be referred to as an associated functional of $C$.

2. The main results about well-capped cones are extended to well-capped sets in this section. In his discussion of locally compact closed convex sets, Klee [5] does not explicitly use the concept of a cap. However, his techniques can be readily adapted to prove that locally compact convex sets containing no lines are wellcapped and that well-capped sets satisfy a Krein-Milman type theorem. This is done in Theorems 2.1 and 2.2 below. It is also proved (Theorem 2.6) that the closed cone in $E \times R$ generated by $X \times\{1\}$ is well-capped if and only if $X$ is a well-capped subset of $E$. Some related results are given by Hinrichen and Bauer in [4] in which they extend the results of Klee [5] to countable projective limits of locally compact convex sets. They also give an analcg to Theorem 2.6 where "well-capped" is replaced by "locally compact".

Definition. A cap $C$ is said to be a universal cap of $X$ is the affine variety $S_{C}$ spanned by $C$ contains $X$.

If $X$ has a universal cap $C$, then $X$ is well-capped. For, if $C$ is extremal, then $X=S_{C} \cap X=C$ (by 1.1(i)). Otherwise let $f$ be an associated functional on $S_{C}$. Since $X \subset S_{C}$ each point of $X$ is contained in some $C_{r}=\left\{x \in S_{C} \cap X \mid f(x) \leqq r\right\}$ and hence $X$ is well-capped.

THEOREM 2.1. If $X$ is a locally compact closed convex subset of the LCTVS E such that $X$ contains no line, then $X$ has a universal cap.

Proof. Assume $0 \in X$. By Proposition 3.2 of [5] there is a continuous linear function $f$ on $E$ such that $C_{r}=\{x \in X \mid f(x) \leqq r\}$ is compact for every real number $r$. Since $f$ is linear and defined on all of $E, C_{r}$, with $r$ sufficiently large, is clearly a universal cap of $X$.

THEOREM 2.2. If $X$ is a closed convex well-capped subset of a LCTVS E, then $X$ is the closed convex hull of its extreme points and extremal rays.

Let ext $(X)$ denote the set of extreme points of $X$ and extr $(X)$ the union of extremal rays of $X$. The proof of 2.2 rests on the following simple lemma:

LEMMA 2.3. Let $X$ be a closed convex set and let $f$ be an affine functional on $X$ such that $x_{0} \in \operatorname{ext}\{x \in X \mid f(x)=r\}$. Then $x_{0}$ lies on an extremal line segment or an extremal ray.

Proof. (See also Theorem 3.4 of [5].) If $x_{0} \notin \operatorname{ext}(X)$, then $x_{0}=(y+z) / 2, y$, $z \in X \backslash\left\{x_{0}\right\}$. Since $x_{0} \in \operatorname{ext}\left(f^{-1}(r) \cap X\right)$ it can be assumed that $f(y)<r$ and $f(z)>r$. If [)$y, z(] \cap X$ is not extremal there exist $y^{\prime}, z^{\prime} \in X \backslash D y, z(]$ such that $x_{0} \in\left(y^{\prime}, z^{\prime}\right)$. 
Assume $f\left(y^{\prime}\right)<r$ and $f\left(z^{\prime}\right)>r$. Then there exist $x_{1}, x_{2} \in f^{-1}(r) \cap X$ such that $x_{1} \in\left(y, z^{\prime}\right), x_{2} \in\left(y^{\prime}, z\right)$ and $x_{0} \in\left(x_{1}, x_{2}\right)$. But then $x_{1}=x_{0}=x_{2}$ and hence $y^{\prime}, z^{\prime} \in$ D $y, z$ (] $\cap X$.

Proof of 2.2. Observe first that if $Y$ is a closed convex set which contains a cap $C$, then $\operatorname{ext}(Y) \neq \phi$. For, by 1.6 (iv) either $C$ is extremal or $C$ contains an extremal cap of $Y$. Thus by the Krein-Milman theorem, ext $(Y) \neq \phi$.

Let $X^{\prime}=$ cl-conv $(\operatorname{ext}(X) \cup \operatorname{extr}(X))$ and suppose $x \in X \backslash X^{\prime}$. An application of the separation theorem yields a continuous linear functional $f$ on $E$ such that $f(x)=\alpha<\inf f\left(X^{\prime}\right)$. If $Y=f^{-1}(\alpha) \cap X$, then $Y$ is a closed convex subset of $X$ and hence is well-capped. Let $x_{0} \in \operatorname{ext}(Y)$. By Lemma 2.3 there exist $y, z \in X$ such that $x_{0} \in(y, z)$ and [)$y, z(] \cap X$ is an extremal subset of $X$. Since $X$ is well-capped it contains no lines and thus $[y, z(] \cap X$ is either a point, a closed line segment or a ray. In any case $x_{0} \in \operatorname{cl-conv}(\operatorname{ext} X \cup \operatorname{extr} X)=X^{\prime}$. But $f\left(x_{0}\right)<\inf f\left(X^{\prime}\right)$. Thus it must be the case that $X=X^{\prime}$.

Definition. If $X$ is a convex subset of $E$ let $P(X)$ (or $P$, if no confusion results) denote the closed cone in $E \times R$ generated by $X \times\{1\}$. It is easily seen that $P(X)$ is convex.

Proposition 2.4. If $0<r<s<\infty$ and $U$ is an open set in $E$, then $(r, s) \cdot[U \times\{1\}]$ $=\{(t u, t) \mid r<t<s, u \in U\}$ is open in $E \times R$.

Proof. Let $R^{+}=\{r \in R \mid r>0\}$. The map $F: E \times R^{+} \rightarrow E \times R^{+}$, defined by $F(x, r)=(r x, r)$ is a homeomorphism of $E \times R^{+}$onto itself, taking the open set $U \times(r, s)$ onto $(r, s) \cdot[U \times\{1\}]$.

Definition (Choquet [1]). Let $x_{0} \in X$, where $X$ is a closed convex set. The asymptotic cone $A(X)$ of $X$ is $\bigcup\left\{\left[0, y\left(\mid x_{0}+[0, y(\subset X\}\right.\right.\right.$. It is easily seen that $A(X)$ is a closed convex cone such that $X+A(X) \subset X$. In particular, $A(X)$ is independent of the choice of $x_{0} \in X$.

Proposition 2.5. If $X$ is a closed convex set then $(x, r) \in P(X)$ if and only if either

(i) $r>0$ and $x / r \in X$, or

(ii) $r=0$ and $x \in A(X)$.

Proof. If $r>0$ and $x / r \in X$, then $(x, r)=r(x / r, 1) \subset r \cdot(X \times\{1\}) \subset P$. If $r=0$ and $x \in A$, then $\left(\lambda x_{0}+(1-\lambda) x, \lambda\right)=\lambda\left(\left[x_{0}+(1-\lambda) / \lambda\right] x, 1\right) \subset P$, where $x_{0} \in X$. Since $P$ is closed, letting $\lambda \rightarrow 0$, it is seen that $(x, 0) \in P$.

Now suppose neither condition holds for $(x, r)$ and let $r \geqq 0$. Let $P^{\prime}$ denote the (not necessarily closed) cone generated by $X \times\{1\}$. If $r>0$ and $x / r \notin X$ there is a neighborhood $U$ of $x / r$ in $E$ such that $U \cap X=\phi$. Thus $(s, t) \cdot[U \times\{1\}], 0<s<1$ $<t$, is an open neighborhood of $(x / r, 1)$ in $E \times R$ disjoint from $P^{\prime}$. Thus $(x, r) \notin P$.

Finally, we show that if $(x, 0) \in P$ then $x \in A$. If $x_{0} \in X$ then for any $r \geqq 0$, $\left(x_{0}+r x, 1\right)=\left(x_{0}, 1\right)+r(x, 0) \in P$. By (i) this implies $x_{0}+r x \in X$ and hence $x \in A$. 
Observe that the map $\Phi: E \times R \rightarrow E \times R$ defined by $\Phi(x, t)=\left(x+t x_{0}, t\right)$ is an isomorphism of $E \times R$ onto itself taking $P(X)$ onto $P\left(X+x_{0}\right)$, where $X$ is any closed convex subset of $E$ and $x_{0} \in E$. Consequently translating $X$ in $E$ does not change any essential properties of $P(X)$ (in particular $C$ is a cap of $P(X)$ if and only if $\Phi(C)$ is a cap of $\left.P\left(X+x_{0}\right)\right)$. Thus the location of the origin in $E$ can be chosen for the greatest convenience.

THEOREM 2.6. If $X$ is a closed convex subset of a LCTVS E then $P(X)$ is wellcapped if and only if $X$ is well-capped. Also, $P(X)$ has a universal cap if and only if $X$ has a universal cap.

Proof. If $P$ is well-capped, then $X$, a closed convex subset of $P$, is also wellcapped. Suppose $X$ is well-capped and let $\left(x_{0}, r_{0}\right) \in P$. If $r_{0}=0$ then $\left(x_{0}, 0\right) \in A(X)$ $\times\{0\}$. Since $A(X) \times\{0\}$ is a well-capped extremal subset of $P$, Proposition 1.1 shows $\left(x_{0}, 0\right)$ is contained in a cap of $P$. If $r_{0}>0$ it suffices to find a cap $C$ of $P$ containing $\left(x_{0} / r_{0}, 1\right)$. Thus, assume to begin with that $r_{0}=1$. In this case $x_{0} \in X$ so that there is a cap $C_{1}$ of $X$ containing $x_{0}$. Using the observation preceding the theorem it can be assumed the origin is situated as follows: If $C_{1}$ is extremal, let $0 \in C_{1}$. Otherwise let $f$ be an associated functional on the affine variety $S\left(C_{1}\right)$ spanned by $C_{1}$ and let $0 \in\left\{x \in C_{1} \mid f(x)=\inf f\left(C_{1}\right)\right\}$. Thus $f$ can be chosen to be linear and bounded below by 0 on $S\left(C_{1}\right) \cap X$. In either case $S\left(C_{1}\right)$ is a linear subspace. By 1.1, $S\left(C_{1}\right) \cap X$ is an extremal subset of $X$. Let $S=\left(S\left(C_{1}\right) \times R\right) \cap P(X)$. It is not difficult to show $S$ is an extremal subset of $P(X)$ containing $C_{1} \times\{1\}$.

Suppose now $C_{1}$ is an extremal cap of $X$. Then $S=P\left(C_{1}\right)=[0, \infty)\left[C_{1} \times\{1\}\right]$ and $C=[0,1]\left[C_{1} \times\{1\}\right]$ is a cap of $P\left(C_{1}\right)$ containing $C_{1} \times\{1\}$. Since $P\left(C_{1}\right)$ is extremal in $P, C$ is a cap of $P$. If $C_{1}$ is a universal extremal cap, then $C_{1}=X$ and $C$ is a universal cap of $P$. If $C_{1}$ is not extremal in $X$, then define $F$ on $S\left(C_{1}\right) \times R$ by $F(x, r)$ $=(f(x)+r) / 2$, where $f$ is the associated functional of $C_{1}$, and let

$$
C=\{(x, r) \in S \mid F(x, r) \leqq 1\} .
$$

Clearly $C_{1} \times\{1\} \subset C$. Since $F$ is linear on $S\left(C_{1}\right) \times R$ and $S=\left[S\left(C_{1}\right) \times R\right] \cap P$ is extremal it follows from 1.1 that $C$ is a cap, providing $C$ is compact. Since $f$ is l.s.c. on $S\left(C_{1}\right) \cap X, f$ is 1.s.c. on $S\left(C_{1}\right) \cap 2 X$. Thus $f$ is 1.s.c. on $2 C_{1}$ so that $F$ is 1.s.c. on the compact set $K=\left(2 C_{1} \times[0,2]\right) \cap P \subset S$. Let $C^{\prime}=\{(x, r) \in K \mid F(x, r) \leqq 1\}$. We will show $C=C^{\prime}$ thus proving $C$ is compact. Clearly $C^{\prime} \subset C$. Suppose $(x, r) \in C$. Since $F(x, r)=(f(x)+r) / 2 \leqq 1$ and $f(x) \geqq 0,0 \leqq r \leqq 2$. If $r=0$ then $x \in A$. Since $f(x) \leqq 2$, $x \in C_{2} \cap A \subset 2 C_{1}$, where $C_{2} \doteq\left\{x \in S\left(C_{1}\right) \cap X \mid f(x) \leqq 2\right\}$. Suppose $r>0$. Now $f(x / r) \leqq(2-r) / r$ so that $x / r \in C_{(2-r) / r}$. If $0<r \leqq 1$ then $x \in r C_{(2-r) / r} \subset C_{2-r} \subset C_{2}$ $\subset 2 C_{1}$. If $1 \leqq r \leqq 2$ then $x \in r C_{(2-r) / r} \subset r C_{1} \subset 2 C_{1}$. Thus $(x, r) \in C^{\prime}$ and so $C=C^{\prime}$.

If $C_{1}$ is a universal cap of $X$ then $S=P(X)$ and it follows from the definition of $C$ that $C$ is a universal cap of $P(X)$. Also, if $C$ is any universal cap of $P(X)$, then $r C \cap[X \times\{1\}]$ is a universal cap of $X$, for $r$ sufficiently large. 
Choquet has shown (see also [7] and [8]) that if $E=\prod_{n=1}^{\infty} E_{n}$, where $E_{n}$ is a LCTVS and $P_{n}$ is a well-capped cone in $E_{n}$, then $P=\prod_{n=1}^{\infty} P_{n}$ is a well-capped cone in $E$. The analogous result for well-capped sets can be stated as a corollary to this and to Theorem 2.6.

COROLlARY 2.7. If $X_{n} \subset E_{n}$ is a closed convex well-capped set for each positive integer $n$, then $X=\prod_{n=1}^{\infty} X_{n} \subset E=\prod_{n=1}^{\infty} E_{n}$ is a well-capped subset of $E$.

3. The most frequently encountered examples of well-capped cones are cones of positive measures on locally compact Hausdorff spaces. In this section the caps of various positive cones of measures are discussed.

Let $T$ denote a locally compact Hausdorff space and let $C_{0}(T)$ be the space of all continuous real-valued functions on $T$ which "vanish at infinity". That is, $C_{0}(T)$ consists of those $f$ with the property that for each $\varepsilon>0$ there exists a compact subset $B$ of $T$ such that $x \notin B$ implies $|f(x)|<\varepsilon$. Then $C_{0}(T)$ is a Banach space in the sup norm and its dual $C_{0}(T)^{*}$ can be identified with the space of all finite regular Borel measures on $T$.

THEOREM 3.1. If $T$ is a locally compact Hausdorff space, then the positive cone $P$ of $C_{0}(T)^{*}$ has a universal cap in the weak* topology.

Proof. Let $U$ denote the unit ball in $C_{0}(T)^{*}$. Let $C=\{\mu \in P \mid \mu(T)=\|\mu\| \leqq 1\}$ $=U \cap P$. The set $C$ is a universal cap of $P$.

Let $C(T)$ be the space of all continuous real-valued functions on $T$ with the topology of uniform convergence on compacta. The dual $C(T)^{*}$ can be identified with the space of all regular Borel measures with compact support [3]. The support of a positive measure $\mu$ is taken to mean $\bigcap\{F \subset T \mid F$ closed and $\mu(T \mid F)=0\}$. The support of a signed measure is the support of its total variation. The measures in $C(T)^{*}$ are finite since they have compact support.

THEOREM 3.2. If $T$ is a locally compact Hausdorff space, then the positive cone $P$ of $C(T)^{*}$ is well-capped in the weak* topology.

Proof. Let $B$ be a compact subset of $T$ and let $r$ be a positive real number. Then $P^{\prime}=\{\mu \in P \mid$ supp $\mu \subset B\}$ is an extremal subset of $P$. Let $C=\left\{\mu \in P^{\prime} \mid \mu(T) \leqq r\right\}$. Let $U=\left\{f \in C(T)\left|\sup _{x \in B}\right| f(x) \mid \leqq 1 / r\right\}$. Then $U$ is a neighborhood of 0 in $C(T)$ and $C$ is a closed subset of $U^{0}=\{\mu|| \mu(f) \mid \leqq 1$ for all $f \in U\}$. Thus $C$ is compact and therefore a cap of $P$. Since every measure in $P$ is contained in such a cap, $P$ is well-capped.

Consider now $C_{\infty}(T)=\{f \in C(T) \mid$ there exists $B \subset T, B$ compact and supp $f \subset B\}$. One form of the Riesz Representation Theorem states that to each positive linear functional $\phi$ on $C_{\infty}(T)$ there corresponds a unique regular Borel measure $\mu$ on $T$ such that $\phi(f)=\int f d \mu$ for all $f \in C_{\infty}(T)$. Let $P$ be the cone of positive regular Borel measures on $T$.

It is convenient to represent $P$ as a projective limit of well-capped cones of measures. Toward this end let $\mathscr{B}$ be a family of open subsets of $T$ with compact 
closure (directed by inclusion) such that if $C$ is any compact subset of $T$ then $C \subset B$ for some $B \in \mathscr{B}$. Let $E_{B}=C_{0}(B)$ with the norm topology. Then $E_{B}$ is isometrically isomorphic to $\left\{f \in C_{\infty}(T) \mid \operatorname{supp} f \subset \bar{B}\right\}$. Let $E_{B}^{*}$ be the dual of $E_{B}$ with the weak* topology. Then 3.1 shows the positive cone of $E_{B}^{*}$ has a universal cap.

Let $E^{*}=\lim \operatorname{proj}\left\{E_{B}^{*} \mid B \in \mathscr{B}\right\}=\left\{F \in \prod_{B} E_{B}^{*} \mid F\left(B_{1}\right)(f)=F\left(B_{2}\right)(f)\right.$ whenever $B_{1}$ $\subset B_{2}$ and $\left.f \in E_{B_{1}}\right\}$. Then $P$ with the weak topology induced by $C_{\infty}(T)$ is isomorphic to the positive cone of $E^{*}$ with the relative product topology under the correspondence $\mu \rightarrow F_{\mu}$, where $F_{\mu}(B)(f)=\mu(f)=\int f d \mu$. The correspondence is $1-1$ and onto by the Riesz Representation Theorem cited above. It follows easily from the definition of $E^{*}$ that the correspondence is a homeomorphism.

Suppose now that $T$ is locally compact and $\sigma$-compact. Meyer has shown that in this case the cone of positive regular Borel measures is well-capped by constructing a cap containing a given positive measure [7]. An alternative proof, implicit in [1], uses Choquet's theorem that a countable product of well-capped cones is wellcapped.

TheOREM 3.3 (MeYer). Let $T$ be a locally compact, $\sigma$-compact Hausdorff space. Then the cone of positive regular Borel measures on $T$ is well-capped.

Proof. Since $T$ is $\sigma$-compact, the collection $\mathscr{B}$ can be chosen to be countable. The positive cone $P$ of the projective limit $E^{*}$ is easily seen to be a closed subset of the positive cone of $\prod E_{B}^{*}$. Since this product is countable $P$ is well-capped by 2.7 . This shows that the cone of positive regular Borel measures is well-capped.

If $T$ is not $\sigma$-compact then the cone of positive measures need not be well-capped. As an example let $T$ be an uncountable discrete space. It will follow from Theorem 3.5 below that if $\mu$ is any measure on $T$ whose support is not $\sigma$-compact then $\mu$ is not contained in a cap.

However, we can state the following theorem.

THEOREM 3.4. If $\mu_{0}$ is a positive regular Borel measure on the locally compact Hausdorff space $T$, and if either

(a) $\mu_{0}(T)<\infty$, or

(b) supp $\mu_{0}$ is $\sigma$-compact,

then $\mu_{0}$ is contained in a cap of the cone $P$ of positive regular Borel measures on $T$.

Proof. Let $P^{\prime}=\{\mu \in P \mid \mu(T)<\infty\}$. Then $P^{\prime}$ is an extremal subset of $P$. Let $C=\left\{\mu \in P^{\prime} \mid \mu(T) \leqq 1\right\}$. If $U=\left\{f \in C_{\infty}(T)|\sup | f(T) \mid \leqq 1\right\}$ then $U^{0}$ is a compact subset of $P^{\prime}-P^{\prime}$ in the weak topology induced by $C_{\infty}(T)$. But $C$ is a closed subset of $U^{0}$ so that $C$ is a universal cap of $P^{\prime}$. Thus (a) follows.

If supp $\mu_{0}$ is $\sigma$-compact let $T^{\prime}$ be the support of $\mu_{0}$ and let $P^{\prime}=\left\{\mu \in P \mid \operatorname{supp} \mu \subset T^{\prime}\right\}$. Again $P^{\prime}$ is an extremal subset of $P$. Also $P^{\prime}$ is isomorphic to the cone of positive regular Borel measures on $T^{\prime}$ with the weak topology induced by $C_{\infty}\left(T^{\prime}\right)$. Thus by 3.4 $P^{\prime}$ is well-capped and therefore $\mu_{0}$ is contained in a cap of $P$. 
There may be measures on $T$ which satisfy (a) but not (b). As an example consider the space $T$ consisting of an uncountable product of unit intervals with one point of the product removed. Since an uncountable product of intervals is not first countable it follows easily that $T$ is not $\sigma$-compact. If $\mu$ denotes the product of Lebesque measures, restricted to $T$, then $\mu$ is finite and the support of $\mu$ is $T$.

The following theorem shows that this cannot happen if $T$ is paracompact.

THEOREM 3.5. Let $T$ be a paracompact, locally compact Hausdorff space. A measure $\mu$ in the cone $P$ of positive regular Borel measures on $T$ is contained in a cap of $P$ if and only if the support of $\mu$ is $\sigma$-compact.

Proof. We use the fact that a locally compact paracompact space is the union of disjoint open $\sigma$-compact subsets (see, e.g. Theorem 7.3 of [2]). Thus if $X$ is a closed subset of $T$ and $X$ is not $\sigma$-compact then there exists an uncountable family $\mathscr{W}$ of pairwise disjoint open sets such that $W \in \mathscr{W}$ implies $W \cap X \neq \phi$.

Assume $\mu_{0} \in P$ and supp $\mu_{0}=X$ is not $\sigma$-compact. Suppose that $\mu_{0} \in P$ is contained in a cap $C$ of $P$. Let $F$ be the linear functional associated with $C$ (as in 1.6). Let $\mathscr{W}$ be a family of open sets as above. If $W \in \mathscr{W}$ then $W \cap X \neq \phi$ and therefore $\mu_{0}(W)>0$. Let $\mu_{W}=\left.\mu_{0}\right|_{w}$.

Note that $0 \leqq \mu_{W} \leqq \mu_{0}$ implies that $\mu_{W} \in C$. Since $\mu_{W}>0$ and $C$ is compact, $F\left(\mu_{W}\right)>0$. If $W_{1}, \ldots, W_{n} \in \mathscr{W}$, then $\sum_{k=1}^{n} \mu_{W_{k}} \leqq \mu_{0}$. But $F\left(\sum_{k=1}^{n} \mu_{W_{k}}\right)=\sum_{k=1}^{n} F\left(\mu_{W_{k}}\right)$ $\leqq 1$. On the other hand, since $\mathscr{W}$ is uncountable and $F\left(\mu_{W}\right)>0$ for all $W \in \mathscr{W}$, the supremum of the above sums taken over all finite subsets of $\mathscr{W}$ is $+\infty$. This contradiction shows that no cap of $P$ contains $\mu_{0}$.

4. In this section some examples of sets and cones which fail to be well-capped are discussed. It would seem plausible that the vector sum $P+B$ of a well-capped cone $P$ and a compact convex set $B$ is well-capped, but the following example shows that this is not the case.

THeOREM 4.1. There exist a closed convex cone $P$ with a universal cap and a compact convex set $B$ such that the closed convex set $X=P+B$ is not well-capped.

Proof. Let $T$ be an uncountable discrete space and let $E=l^{1}(T)$, with the weak* topology as the dual of $c_{0}(T)$. Let $P$ be the positive cone of $E$ and let $B$ be the unit ball of $E$. By $3.1 P$ has a universal cap and $B$ is a compact convex subset of $E$ in the weak* topology.

Every element $x \in E$ can be written in the form $x=x^{+}-x^{-}$, where $x^{+}(t)=x(t) \vee 0$ and $x^{-}(t)=-(x(t) \wedge 0)$. If $X=P+B$ then $X=\left\{x \in E \mid \sum_{t \in T} x^{-}(t) \leqq 1\right\}$.

We will show now that if $C$ is any cap of $X$ then $C$ contains no nonzero element of $P$. For suppose $x_{0} \neq 0$, and $x_{0} \in C \cap P$. Since $C \cap P$ is a cap of $P, 0 \in C \cap P \subset C$. Let $S_{C}$ be the linear subspace generated by $C$ and let $\delta_{t}(s)=\delta_{t s}$, the Kronecker delta.

For any $t \in T$ we can write $0=r /(1+r)\left[-\delta_{t}\right]+1 /(1+r)\left[r \delta_{t}\right]$ where $r$ is a positive number sufficiently large so that $r \delta_{t} \in X \mid C$. This shows that $-\delta_{t} \in C$ and that 
$\delta_{t} \in S_{C}$. Also the cap boundary $B_{C}$ is not empty. Since $0 \in C \mid L_{C}\left(L_{C}\right.$ is the linear variety generated by $B_{C}$ ) by 1.6 there is a linear functional $f$ on $S_{C}$ such that

$$
\left\{x \in S_{C} \cap X \mid f(x) \leqq 1\right\}=C .
$$

Since $C$ contains no rays $f$ must be strictly positive on (the nonzero elements of) $P \cap S_{C}$. Let $a_{t}=f\left(\delta_{t}\right)$. Then $a_{t}>0$ for all $t \in T$. We show next that $f(x)=\sum_{t \in T} a_{t} x(t)$, for all $x \in S_{C} \cap P$. If $x=\sum_{t \in T^{\prime}} x(t) \delta_{t}$, where $T^{\prime}$ is a finite subset of $T$, then $x \in S_{C}$ and $f(x)=\sum_{t \in T} a_{t} x(t)$. Given any $x \in S_{C} \cap P$ let $y_{T^{\prime}}=\sum_{t \in T^{\prime}} x(t) \delta_{t}$, where $T^{\prime}$ is finite. Then with $T^{\prime}$ ranging over the finite subsets of $T,\left\{y_{T^{\prime}}\right\}$, is a net in $S_{C} \cap P$ converging to $x$. Since $f$ is 1.s.c. on $S_{C} \cap P, f(x) \leqq \lim \inf f\left(y_{T^{\prime}}\right) \leqq \sum_{t \in T} a_{t} x(t)$. Since $f$ is positive on $P \cap S_{C}$ and $y_{T^{\prime}} \leqq x, f(x) \geqq f\left(y_{T^{\prime}}\right)$, for all $T^{\prime}$. Thus $f(x) \geqq \lim \sup f\left(y_{T^{\prime}}\right)$. This shows that $f(x)=\lim f\left(y_{T^{\prime}}\right)=\sum_{t \in T} a_{t} x(t)$.

Since $T$ is uncountable and each $a_{t}>0$, there exists a positive integer $N$ such that $T^{\prime}=\left\{t \in T \mid a_{t} \geqq 1 / N\right\}$ is uncountable. But now $C$ cannot be closed. For, take $x=\left[(1+\alpha) / a_{t_{0}}\right] \delta_{t_{0}}$ where $0<\alpha<1 / N$ and $t_{0} \in T$. Then $x \in S_{C}$ and $f(x)=1+\alpha>1$ so that $x \notin C$. Let $U=\left\{y \in E|| s_{i}(y)-s_{i}(x) \mid<\varepsilon, i=1, \ldots, n\right\}$, where $s_{1}, \ldots, s_{n} \in c_{0}(T)$. $U$ is a weak* neighborhood of $x$. Now $T^{\prime \prime}=\left\{t \in T \mid s_{i}(t) \neq 0\right.$, some $\left.i \in 1, \ldots, n\right\}$ is countable. Let $t^{\prime} \in T^{\prime} \backslash T^{\prime \prime}$. Let $y=x-\delta_{t^{\prime}}$. Since $s_{i}\left(\delta_{t^{\prime}}\right)=0, i=1, \ldots, n$, we have $s_{i}(y)=s_{i}(x)$ so that $y \in U \cap S_{C}$. But $f(y)=1+\alpha-a_{t^{\prime}} \leqq 1$ so that $y \in C$. But $U$ is an arbitrary neighborhood of $x$ and $U \cap C \neq \phi$. Thus $x \in \bar{C} \mid C$. This shows that no cap of $X$ can contain $x_{0}$.

The positive cones of various $L^{p}$ spaces are now investigated. Consider first the $l^{p}$ spaces, $1 \leqq p \leqq \infty$.

THEOREM 4.2. The positive cone of $l^{p}(1 \leqq p \leqq \infty)$ is well-capped in the weak* topology if and only if $p=1$.

Proof. By 3.1 the positive cone of $l^{1}$ has a universal cap. Suppose now that $1<p \leqq \infty$. Let $a=\left\{a_{n}\right\}_{n=1}^{\infty}$ be a sequence of positive numbers such that $a \in l^{p} \mid l^{1}$. Suppose that $a \in C$, where $C$ is a cap of the positive cone of $l^{p}$. Let $f$ be the associated linear functional and assume without loss of generality that $f(a)=1$. Let $\delta^{n}$ denote the sequence which is 1 in the $n$th place and 0 elsewhere. Since $a_{n} \delta^{n} \leqq a, a_{n} \delta^{n} \in C$. Thus $f\left(a_{n} \delta^{n}\right)=a_{n} f\left(\delta^{n}\right)>0$. Let $b_{n}=f\left(\delta^{n}\right)$. Then $b_{n}>0$. Let $s_{n}=\sum_{k=1}^{n} a_{k} \delta^{k}$. Since $s_{n} \rightarrow a$ in the norm topology it converges to $a$ in the weak* topology. Since $f$ is l.s.c. on $C, f(a) \leqq \lim \inf f\left(s_{n}\right)$. Also $s_{n} \leqq a$, for all $n$, so that $f\left(a-s_{n}\right) \geqq 0$. Thus $f(a)$ $\geqq \lim \sup f\left(s_{n}\right)$ and consequently $f(a)=\lim f\left(s_{n}\right)=\sum_{n=1}^{\infty} a_{n} b_{n}<\infty$. This implies that there exists a subsequence $\left\{b_{n_{k}}\right\}$ of $\left\{b_{n}\right\}$ such that $\lim _{k \rightarrow \infty} b_{n_{k}}=0$. For if not, there exist $\varepsilon>0$ such that $b_{n} \geqq \varepsilon$, for all $n$. In this case $\sum a_{n} b_{n} \geqq \varepsilon \sum a_{n}=+\infty$, since $a \notin l^{1}$. So assume $\left\{b_{n_{k}}\right\}$ has been chosen so that $0<b_{n_{k}} \leqq 1 / k$, for all $k$. Then $f\left(k \delta^{n_{k}}\right)$ $=k b_{n_{k}} \leqq 1$. This implies $k \delta^{n_{k}} \in C$. Since $\left\|k \delta_{n_{k}}\right\|=k, C$ is unbounded in norm. But $l^{p}$ is the dual of a Banach space so that if $C$ is unbounded in norm it is also unbounded in the weak* topology. Thus $C$ is not compact.

We now consider $L^{p}(X, \mathscr{S}, \mu)$ spaces where there exists a nonatomic measurable subset of $X$. 
THEOREM 4.3. Let $(X, \mathscr{S}, \mu)$ be a complete $\sigma$-finite measure space and suppose there exists an $S \in \mathscr{S}$ such that $0<\mu(S)<\infty$ and $S$ contains no atoms with respect to $\mu$. Then the positive cone of $L^{p}(X, \mathscr{S}, \mu), 1<p \leqq \infty$, in the weak* topology as the dual of $L^{q}(X, \mathscr{S}, \mu)(1 / p+1 / q=1)$ is not well-capped.

Proof. We show that no cap of the positive cone $P$ of $L^{p}$ contains $\mathscr{X}_{S}$, (the characteristic function of $S$ ). For suppose $\mathscr{X}_{S} \in C$, where $C$ is a cap of $P$. Let $F$ be the associated linear functional and assume $F\left(\mathscr{X}_{S}\right)=1$. Then $F$ defines a finite measure $\mu_{F}$ on the measurable subsets of $S$ by $\mu_{F}(A)=F\left(\mathscr{X}_{A}\right)$. Since $F$ is positive we have $\mu_{F}(A) \leqq \mu_{F}(B)$ whenever $A \subset B \subset S$. To see that $\mu_{F}$ is countably additive let $A=$ $\bigcup_{n=1}^{\infty} A_{n},\left\{A_{n}\right\}_{n=1}^{\infty}$ a pairwise disjoint collection of measurable subsets of $S$. Let $B_{n}$ $=\bigcup_{k=1}^{n} A_{k}$. Then $\mathscr{X}_{B_{n}} \leqq \mathscr{X}_{A} \leqq \mathscr{X}_{S} \in C$ and $\mathscr{X}_{B_{n}} \rightarrow \mathscr{X}_{A}$ in the weak* topology. Since $F$ is a positive functional 1.s.c. on $C$, we have $\mu_{F}(A)=\lim F\left(\mathscr{X}_{B_{n}}\right)=\sum_{n=1}^{\infty} \mu_{F}\left(A_{n}\right)$.

Since $S$ has no atoms, given a positive integer $n$ we can choose $n$ disjoint subsets $S_{1}, \ldots, S_{n}$ of $S$ such that $\mu\left(S_{k}\right)=\mu(S) / n$, and $S=\bigcup_{k=1}^{n} S_{k}$. (This is by Liapunov's Theorem, see e.g. [6].) Now $1=F\left(\mathscr{X}_{S}\right)=\sum_{k=1}^{n} F\left(\mathscr{X}_{S_{k}}\right)$ so that there exists $S_{k}$ such that $0<F\left(\mathscr{X}_{S_{k}}\right) \leqq I / n$. Then $n \mathscr{X}_{S_{k}} \in C$ since $F\left(n \mathscr{X}_{S_{k}}\right) \leqq 1$. But $\left\|n \mathscr{X}_{S_{k}}\right\|_{p}=\left(\int_{S_{k}} n^{p} d \mu\right)^{1 / p}$ $=\left(n^{p} \mu(S) / n\right)^{1 / p}=[\mu(S)]^{1 / p}=n^{(p-1) / p}$, for $1<p<\infty$, and $\left\|n \mathscr{X}_{S_{k}}\right\|_{\infty}=n$. Thus $C$ is unbounded in norm and since $L^{p}$ is the dual of a Banach space $C$ is unbounded in the weak* topology and hence not compact.

THEOREM 4.4. Let $T$ be a completely regular Hausdorff space and let $E=C(T)$, the space of all continuous real-valued functions on $T$. Then the positive cone $P$ of $E$ is well-capped in the topology of pointwise convergence if and only if $T$ is countable and discrete.

Proof. Suppose $P$ is well-capped. Given $x \in T$ choose an open neighborhood $U_{x}$ of $x$ and choose $f \in P$ such that $f(T) \subset[0,1], f(x)=1$ and $f\left(T \mid U_{x}\right)=\{0\}$. Let $C$ be a cap of $P$ containing $f$. For each open neighborhood $U$ of $x, U \subset U_{x}$, there exists $g \in E$ such that $g(T) \subset[0,1], g(x)=1$ and $g(T \mid U)=\{0\}$. Let $f_{U}=f \wedge g$. Then $0<f_{U} \leqq f$ so that $f_{U} \in C$. With the open neighborhoods of $x$ directed down by inclusion $\left\{f_{U}\right\}$ is a net in $C$ converging pointwise to $\delta_{x}$, the characteristic function of $\{x\}$. Since $C$ is compact, $\delta_{x} \in C \subset E$. But $\delta_{x}$ continuous implies $\{x\}$ is open in $T$. Since this applies to any $x, T$ is discrete. Thus $T$ is locally compact and $P$ can be identified with the cone of positive regular Borel measures on $T$. But the fact that $T$ is discrete means, in particular, that $T$ is paracompact and by $3.5 P$ is well-capped if and only if $T$ is $\sigma$-compact. But $T$ is $\sigma$-compact if and only if $T$ is countable.

If $T$ is assumed to be locally compact and Hausdorff, then the same argument can be applied to $C_{\infty}(T)$.

THEOREM 4.5. If $T$ is a locally compact Hausdorff space, then the positive cone $P$ of $C_{\infty}(T)$ is well-capped in the topology of pointwise convergence if and only if $T$ is discrete. 
Proof. As in 4.4, if $P$ is well-capped then $T$ is discrete. But in this case, $P$ can be identified with the cone of positive regular Borel measures with compact support. Then $P$ is well-capped by 3.2 .

Observe that if the positive cone of $C(T)$ or $C_{\infty}(T)$ fails to be well-capped in the topology of pointwise convergence, then it is certainly not well-capped in any stronger topology.

\section{REFERENCES}

1. Gustave Choquet, Les cônes convexes faiblement complets dans l'analyse, Proc. Internat. Congress Math. Stockholm, 1962, pp. 317-330.

2. J. Dugundji, Topology, Allyn and Bacon, Boston, 1966.

3. R. E. Edwards, Functional analysis, Holt, New York, 1965.

4. D. Hinrichen and H. Bauer, Einige Eigenschaften lokal-kompakter konvexer Megnen und ihrer projectiven Limiten, Proc. Colloq. Convexity, Copenhagen, 1965.

5. V. L. Klee, Jr., Extremal structure of convex sets, Arch. Math. 8 (1957), 234-240.

6. Joram Lindenstrauss, A short proof of Liapounov's convexity theorem, J. Math. Mech. 15 (1966), 971-973.

7. P. A. Meyer, Theory of probability, Blaisdell, New York, 1965.

8. R. R. Phelps, Lectures on Choquet's theorem, Van Nostrand, Princeton, N. J., 1966.

UNIVERSITY OF WASHINGTON,

SeattLe, Washington

UNIVERSITY OF CALIFORNIA,

Berkeley, CALIFornia 